\title{
Gasser-Leutwyler coefficients: A progress report
}

\author{
George T. Fleming ${ }^{\mathrm{a}}$, Daniel R. Nelson ${ }^{\mathrm{a}}$ and Gregory W. Kilcup ${ }^{\mathrm{a}}$ \\ a Physics Department, The Ohio State University, Columbus, OH 43210-1168, USA.
}

Last year, we reported our first results on the determination of Gasser-Leutwyler coefficients using partially quenched lattice QCD with three flavors of dynamical staggered quarks. We give an update on our progress in determining two of these coefficients, including an exhaustive effort to estimate all sources of systematic error. At this conference, we have heard about algorithmic techniques to reduce staggered flavor symmetry breaking and a method to incorporate staggered flavor breaking into the partially quenched chiral Lagrangian. We comment on our plans to integrate these developments into our ongoing program.

\section{Introduction}

The broad application of effective field theories like the chiral Lagrangian to the description of low energy phenomena in QCD has been one of the major theoretical advances of the last quarter century [1]. One substantial limitation of this approach is that the chiral Lagrangian has additional symmetry [2] that precludes the determination of all the low energy constants (LEC's) of the effective theory using only experimental input. This problem is resolved by computing low energy observables directly in QCD and fixing the undetermined LEC's by matching to predictions of chiral perturbation theory (ChPT).

Unfortunately, the low energy regime of $\mathrm{QCD}$ is non-perturbative, so relevant observables are not so easily computed. This has led to an entire industry of estimating LEC's (with uncontrolled systematic errors) using model calculations and phenomenology, culminating in a generally accepted set of values [3]. Lattice QCD has also made some inroads in this area, with its own set of systematic errors, including errors due to $N_{f} \neq 3$ and dynamical quarks that are too heavy. See Wittig's review talk for a current summary [4].

Recently it was realized that $\mathrm{ChPT}$ and the corresponding partially quenched chiral perturbation theory (pqChPT) 河,6] share the same low energy constants [7,8]. As partially quenched QCD (pqQCD) simulations are somewhat less computationally demanding, recent efforts have been made to determine the LEC's, particularly those affecting the mass of the up quark [9]. However, as the number of light dynamical sea quarks $N_{f}$ doesn't appear explicitly in the chiral Lagrangian, LEC's computed with $N_{f} \neq 3$ have no $a$ priori relation to those of phenomenological interest, although a posteriori evidence suggests that the $N_{f}$ dependence may be mild. Here, we will review our recent $N_{f}=3$ calculations 10, emphasizing our program to estimate all expected systematic errors, and discuss our plans for reducing the systematic errors in the future.

\section{LEC's and systematic error}

Our goal is to determine the low energy constants $2 \alpha_{8}-\alpha_{5}$ and $2 \alpha_{6}-\alpha_{4} 1$ by studying the sea and valence quark mass $\left(m_{S}\right.$ and $\left.m_{V}\right)$ dependence of the pseudo-Goldstone meson mass $\widetilde{M}_{\pi}$ and decay constant $f_{\pi}$. NLO pqChPT predicts the dependence in terms of the LEC's, with loops cut off at $\Lambda_{\chi} \equiv 4 \pi f$, as

$$
\begin{aligned}
\widetilde{M}_{\pi}^{2}= & z \Lambda_{\chi}^{2} m_{V}\left[1+z m_{V}\left(2 \alpha_{8}-\alpha_{5}+\frac{1}{N_{f}}\right)\right. \\
& +2 m_{S} N_{f}\left(2 \alpha_{6}-\alpha_{4}-\frac{1}{N_{f}^{2}}\right) \\
& \left.+\frac{z}{N_{f}}\left(2 m_{V}-m_{S}\right) \log \left(m_{V}+m_{S}\right)\right]
\end{aligned}
$$

\footnotetext{
${ }^{1} \alpha_{i} \equiv 8(4 \pi)^{2} L_{i}$
} 


$$
\begin{aligned}
f_{\pi}= & f\left[1+\frac{\alpha_{5}}{2} z m_{V}+\frac{\alpha_{4}}{2} z m_{S} N_{f}\right. \\
& \left.+\frac{z N_{f}}{4}\left(m_{V}+m_{S}\right) \log \frac{z}{2}\left(m_{V}+m_{S}\right)\right](2)
\end{aligned}
$$

where $z \equiv 2 \mu / \Lambda_{\chi}^{2}$ is related to the chiral condensate $\mu$ and $\widetilde{M}$ refers to a QCD meson mass, without QED contributions. Similar formulae for fixed $m_{S}$ can be used to extract only $\alpha_{5}$ and $2 \alpha_{8}-\alpha_{5}$. We refer readers first to our earlier work [10] and focus on details not covered there.

The primary sources of systematic error are finite volume, finite lattice spacing, finite step size, flavor (or "taste" [11]) symmetry violations and the choice of sea and valence quark masses for observables included in the fitting procedure. The errors are not independent. For example, as smaller quark masses are included in the analysis, larger volumes are required to minimize finite volume errors. Also, at smaller lattice spacings all systematic errors due to lattice artifacts are reduced, including flavor symmetry violations. So, while not justified by the preceding observation, we have chosen to make generous estimates of our systematic errors and add them in quadrature.

Our procedure for estimating systematic errors is simple. Holding all other parameters fixed, we independently vary the volume, step size, lattice spacing and sea quark mass. To estimate fitting systematics, we vary the range of quark masses included in the fit, ensuring that all masses are within the expected radius of convergence of the effective theory. To estimate flavor symmetry breaking still remaining at a given lattice spacing, we perform HYP blocking [12] of the configurations and recompute the observables. This procedure may seem ad hoc but it certainly has the correct continuum limit as the blocking becomes irrelevant for sufficiently smooth gauge fields.

As our paper shows, this recipe for estimating systematic errors works well with $\widetilde{M}_{\pi}$. HYP blocking doesn't significantly change the leading order behavior, as expected since local blocking shouldn't effect quantities measured at long distances on the lattice. In the presence of flavor breaking, the pions contributing to the chiral logs are too heavy and systematically shift NLO terms away from their continuum values. With HYP blocking, these pions become more degenerate and the systematic error is reduced.

An interesting contrast is what happens to $f_{\pi}$. Since the dominant contributions are local, Fig. 1] clearly shows the effect of HYP blocking on the leading order behavior of $f_{\pi}$. This seems dis-

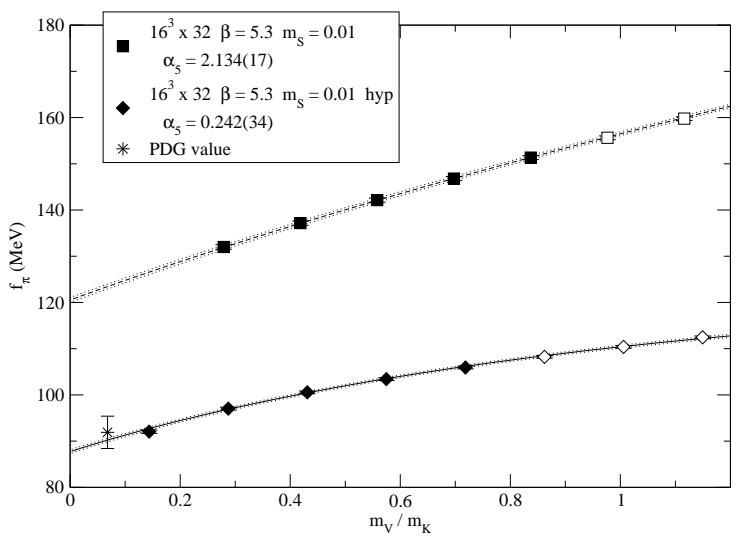

Figure 1. $f_{\pi}$ with and without HYP blocking on $16^{3} \times 32, \beta=5.3$, and $m_{S}=0.01$.

concerting at first glance until one notices the unimproved staggered $f_{\pi}$ is substantially higher than the PDG value whereas the HYP blocked $f_{\pi}$ is consistent with it. The real difficulty comes when extracting $\alpha_{5}$ : for unimproved staggered $\alpha_{5}=2.134(17)$ and for HYP $\alpha_{5}=0.242(34)$. It doesn't seem reasonable to treat the difference in the central values as an estimate of systematic error. Luckily, $\alpha_{5}$ is not affected by the Kaplan-Manohar ambiguity so phenomenological estimates are likely to be more reliable. On the other hand, these estimates currently have $20 \%$ errors so we would like to consider how to better determine LEC's like $\alpha_{5}$ in the next section.

\section{Future plans}

Our original calculation 10] centered around a simulation of a single dynamical sea quark mass at $a^{-1} \approx 1.3 \mathrm{GeV}$, thus it was not possible to extract $2 \alpha_{6}-\alpha_{4}$. However, our study included supporting simulations with several different sea quark masses at $a^{-1} \approx 0.7 \mathrm{GeV}$ on comparable 
physical volumes. So, we offer Fig. 3 as a demonstration of the technique to extract $2 \alpha_{6}-\alpha_{4}$ and $2 \alpha_{8}-\alpha_{5}$ simultaneously. This figure shows a sin-

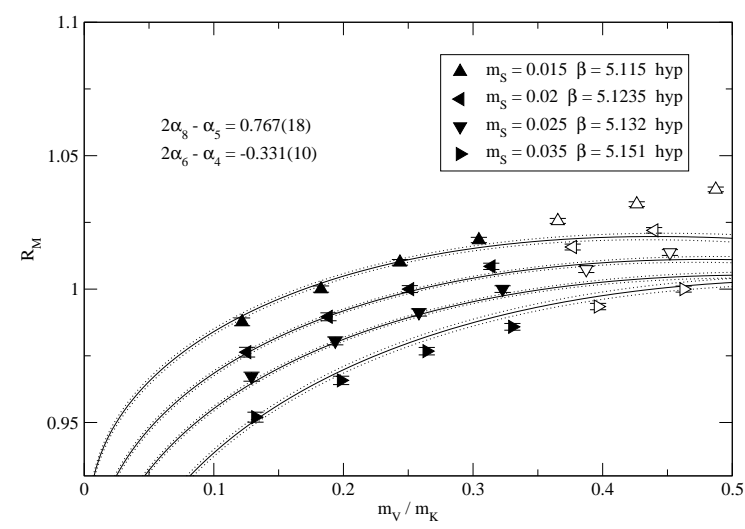

Figure 2. $\quad R_{M} \equiv \widetilde{M}_{\pi}^{2}\left(m_{S}\right) m_{V} / \widetilde{M}_{\pi}^{2}\left(m_{V}\right) m_{S}$ on $8^{3} \times 32$ lattices at $a^{-1} \approx 0.7 \mathrm{GeV}$

gle fit to four simulations with $\chi^{2} /$ dof $\sim 1$ and should be compared to the corresponding figure in 110 where each simulation is fit independently. We expect to perform a detailed extraction of $2 \alpha_{6}-\alpha_{4}$ by this method, including a study of systematic errors, when sufficient data becomes available at smaller lattice spacings.

In section 2, we argued that HYP blocking is useful for estimating systematic errors due to flavor symmetry breaking. At this conference, we have heard about recent efforts to find an efficient dynamical HYP algorithm 13,14. We hope to generate an ensemble of $N_{f}=3$ dynamical HYP lattices which will be used to calculate kaon weak matrix elements for $\epsilon^{\prime} / \epsilon$ [15] as well as GasserLeutwyler coefficients. This should help resolve such questions as how to estimate the systematics of flavor breaking in quantities like $f_{\pi}$.

Also at this conference, Bernard provided an update on his program to incorporate flavor symmetry breaking into ChPT [11]. This is an important development for our program. It altogether eliminates flavor breaking as a source of systematic error as the effects are correctly modeled in our fitting function. It may also increase the range over which the data are well represented by our fitting functions (still within the expected radius of convergence, of course).

Finally, we note that while our determination of $2 \alpha_{8}-\alpha_{5}$ is completely dominated by systematic error, its systematic error is not the dominant source of uncertainty in the ratio $m_{u} / m_{d}$. The dominant uncertainty comes from the unknown electromagnetic contributions to the masses of the pseudoscalar mesons. A technique was devised to measure these effects in quenched lattice simulations [16] and was presented at Lattice '96 [17]. We intend to explore the extension of this work to simulations with dynamical fermions and hope to present our first results at Lattice '03.

\section{REFERENCES}

1. J. Gasser and H. Leutwyler, Nucl. Phys. B250 (1985) 465.

2. D.B. Kaplan and A.V. Manohar, Phys. Rev. Lett. 56 (1986) 2004.

3. J. Bijnens, G. Ecker and J. Gasser, hep$\mathrm{ph} / 9411232$.

4. H. Wittig, Lattice 2002.

5. C.W. Bernard and M.F.L. Golterman, Phys. Rev. D46 (1992) 853, hep-lat/9204007.

6. C.W. Bernard and M.F.L. Golterman, Phys. Rev. D49 (1994) 486, hep-lat/9306005.

7. S.R. Sharpe and N. Shoresh, Phys. Rev. D62 (2000) 094503, hep-lat/0006017.

8. A.G. Cohen, D.B. Kaplan and A.E. Nelson, JHEP 11 (1999) 027, hep-lat/9909091.

9. UKQCD, A.C. Irving et al., Phys. Lett. B518 (2001) 243, hep-lat/0107023.

10. D.R. Nelson, G.T. Fleming and G.W. Kilcup, hep-lat/0112029.

11. C. Aubin et al., hep-lat/0209066.

12. A. Hasenfratz and F. Knechtli, Phys. Rev. D64 (2001) 034504, hep-lat/0103029.

13. A. Alexandru and A. Hasenfratz, heplat/0209070.

14. A. Hasenfratz and A. Alexandru, heplat/0209071.

15. T. Bhattacharya et al., hep-lat/0208050.

16. A. Duncan, E. Eichten and H. Thacker, Phys. Rev. Lett. 76 (1996) 3894.

17. A. Duncan, E. Eichten and H. Thacker, Nucl. Phys. Proc. Suppl. 53 (1997) 295. 